\title{
Caso clínico: Hipersensibilidade tardia à budesonida inalada
}

Case Report: Late hypersensitivity to inhaled budesonide

Rev Port Imunoalergologia $2020 ; 28$ (I): 5 I-55

Sara Carvalho', Guilherme Oliveira² ${ }^{2}$ Fátima Cabral Duarte', Manuel Pereira Barbosa ${ }^{1,3}$

' Serviço de Imunoalergologia, Hospital de Santa Maria, Centro Hospitalar Universitário Lisboa Norte

2 USF Rodrigues Miguéis, ACES Lisboa Norte

${ }^{3}$ Clínica Universitária de Imunoalergologia, Faculdade de Medicina da Universidade de Lisboa

\section{RESUMO}

Introdução: A budesonida é um glucocorticoide usado no tratamento de várias patologias, como asma e rinite. As reações de hipersensibilidade à budesonida são pouco frequentes e na sua maioria são reações tardias. Caso clínico: Doente do sexo feminino, 59 anos, iniciou terapêutica inalatória com budesonida $160 \mathrm{mcg}+$ formoterol $4,5 \mathrm{mcg}$ em contexto de quadro de tosse e pieira recorrente com 6 meses de evolução. Uma semana após o início deste tratamento, apresentou dispneia e ardor orofaríngeo de agravamento progressivo seguido de edema da úvula. Posteriormente, foi encaminhada para a consulta de imunoalergologia. Do estudo realizado, salientam-se testes epicutâneos com a bateria standard do Grupo Português de Dermatites de Contacto positivos para a budesonida (0,1\%) e com produtos da doente, apenas positivos para a associação budesonida $160 \mathrm{mcg}+$ formoterol $4,5 \mathrm{mcg}$, ambos às $96 \mathrm{~h}$. Discussão/Conclusão: Foi, assim, estabelecido o diagnóstico de reação de hipersensibilidade tardia à budesonida inalada, tendo os testes epicutâneos confirmado hipersensibilidade tipo IV.

Palavras-chave: Asma brônquica, budesonida, hipersensibilidade tipo IV, rinite alérgica, testes epicutâneos.

\section{ABSTRACT}

Background: Budesonide is a glucocorticoid used in the treatment of various pathologies such as asthma and rhinitis. Hypersensitivity reactions to budesonide are uncommon and are mostly late reactions. Clinical case: Female patient, 59 years old, started inhaled therapy with budesonide $160 \mathrm{mcg}+$ formoterol $4.5 \mathrm{mcg}$ in a context of recurrent cough and wheezing with 
6 months of evolution. A week after the onset of this treatment, the patient presented with bronchospasm, oropharyngeal burning of progressive aggravation followed by edema of the uvula. Later, she was referred for immunoallergology consultation. From the allergology study, epicutaneous tests with the standard Portuguese Group of Contact Dermatitis were positive for budesonide (0.1\%) and tests with patient's products were positive for budesonide $160 \mathrm{mcg}+$ formoterol $4.5 \mathrm{mcg}$, both at $96 \mathrm{~h}$. Discussion/ Conclusion: The diagnosis of delayed hypersensitivity reaction to inhaled budesonide was established and epicutaneous tests confirmed type IV hypersensitivity.

Keywords: Allergic rhinitis, bronchial asthma, budesonide, hypersensitivity type IV, patch tests.

\section{INTRODUÇÃO}

A budesonida é um glucocorticoide utilizado no tratamento de várias patologias, nomeadamente asma, doença pulmonar obstrutiva crónica, rinite alérgica e outras doenças do foro respiratório e cutâneas ${ }^{1,2}$.

Os corticosteroides podem ser divididos em 4 classes (Quadro I) com base na semelhança da estrutura química. Há um aumento de probabilidade de reatividade cruzada dentro da mesma classe, mas também pode ocorrer entre classes diferentes e interindividualmente'.

Os corticosteroides podem causar um grande espetro de reações adversas, quer em termos de manifestações clínicas quer de mecanismos fisiopatológicos'. Estas reações podem ser não alérgicas ou alérgicas. Do ponto de vista clínico, estas últimas podem ser classificadas como imediatas (surgindo dentro de I hora), habitualmente são lgE-mediadas e caracterizadas mais frequentemente pela presença de urticária, sintomas respiratórios, cardiovasculares, podendo chegar à anafilaxia; e não imediatas (mais de I hora após), mais frequentemente não IgE-mediadas e em que são referidos episódios de exantema maculopapular e urticária tardial-5. As reações de hipersensibilidade aos corticosteroides são na sua maioria reações tardias ${ }^{1-5}$.

O uso extensivo de corticosteróides tópicos tem vindo a ser cada vez mais frequentemente associado ao aumento da prevalência dos casos de reações tardias, com uma prevalência estimada de 0,5 a $5 \%$ 1,3,6,7.
Quadro I. Classificação dos corticosteróides conforme a sua semelhança na estrutura química.

\begin{tabular}{|c|l|l|}
\hline \multicolumn{2}{|c|}{ A } & $\begin{array}{l}\text { Hidrocortisona, metilprednisolona, } \\
\text { prednisolona, acetato de prednisolona, } \\
\text { orednisona, acetato de hidrocortisona, pivalato } \\
\text { de tixocortol }\end{array}$ \\
\hline \multirow{2}{*}{ B } & $\begin{array}{l}\text { Acetonido de triamcinolona, } \\
\text { triamcinolona, budesonida, aminonida, } \\
\text { acetonida de fluocinolona, fluocinonida }\end{array}$ \\
\hline \multirow{2}{*}{ C } & $\begin{array}{l}\text { Betametasona, dexametasona, } \\
\text { fluocortolona }\end{array}$ \\
\hline \multirow{2}{*}{ D } & D.I & $\begin{array}{l}\text { Propionato de clobetasol, valerato } \\
\text { de betametasona, dipropionato de } \\
\text { betametasona, furoato de mometasona }\end{array}$ \\
\cline { 2 - 3 } & D.2 & $\begin{array}{l}\text { Butirato de hidrocortisona, aceponato } \\
\text { de metilprednisolona,prednicarbato }\end{array}$ \\
\hline
\end{tabular}

As reações alérgicas causadas por budesonida manifestam-se geralmente por obstrução nasal, prurido local ou inflamação da mucosa nasal quando administrada por via nasal ou tosse seca, dispneia e edema glossofaríngeo quando administrada por via inalatória ${ }^{1,6,8}$.

Os corticosteroides mais frequentemente associados a reações de hipersensibilidade, quer imediatas quer tardias, são a hidrocortisona e a budesonida, associadas a administração tópica, e a metilprednisolona e hidrocortisona a administração sistémica ${ }^{4}$.

As reações de hipersensibilidade à budesonida são geralmente descritas como reações tardias após inalação ou aplicação nasal, levando a assumir que com maior 
probabilidade se devem a um mecanismo mediado por células $T$ - hipersensibilidade de tipo IV'. A positividade dos testes epicutâneos confirma este mecanismo de hipersensibilidade não mediado por IgE, no entanto pode ser de difícil interpretação, dado o efeito anti-inflamatório da molécula de corticosteroide poder inibir a própria reação tardia ${ }^{1,3}$. Assim, segundo a literatura, os testes epicutâneos com corticosteroides são habitualmente realizados com concentrações de $1 \%$, pois assume-se que concentrações mais elevadas poderão induzir resultados falso-negativos devidos à atividade anti-inflamatória destes fármacos, enquanto concentrações mais baixas podem diminuir a sensibilidade dos testes ${ }^{4}$.

\section{CASO CLÍNICO}

Os autores descrevem o caso clínico de uma doente de 59 anos, caucasiana, que apresenta manifestações clínicas de rinite persistente moderada a grave com 10 anos de evolução, medicada e controlada com furoato de fluticasona 27,5mcg de aplicação nasal e levocetirizina 5 mg/dia. A doente fez anteriormente outras terapêuticas de aplicação nasal, que não sabia especificar. Mais recentemente apresentava ainda quadro com 6 meses de evolução de tosse persistente e pieira recorrente, tendo-lhe sido proposta, na consulta de Pneumologia, terapêutica inalatória com a associação de budesonida $160 \mathrm{mcg}$ e formoterol $4,5 \mathrm{mcg}$. Uma semana após o início deste tratamento, cerca de 4 horas após a última toma, apresentou dispneia, ardor orofaríngeo de agravamento progressivo seguido de edema da úvula. Perante este quadro, automedicou-se com prednisolona $20 \mathrm{mg}$ e levocetirizina $5 \mathrm{mg}$, sem melhoria. Posteriormente recorreu ao serviço de urgência do hospital da área de residência, onde fez terapêutica endovenosa (não especificada), com resolução total da sintomatologia cerca de 3 horas depois. Após este episódio, a doente recorreu à consulta de Pneumologia, tendo sido dada indicação para suspender a terapêutica com a associação de budesonida $160 \mathrm{mcg}$ e formoterol $4,5 \mathrm{mcg}$ e ini- ciar, como alternativa, a associação salmeterol $50 \mathrm{mcg}$ e propionato de fluticasona $250 \mathrm{mcg}$, que realizou com bom controlo das queixas respiratórias e sem reações. Foi posteriormente encaminhada para a consulta de Imunoalergologia, para investigação desta reação suspeita de hipersensibilidade. Na consulta de Imunoalergologia realizou testes epicutâneos com a série básica do Grupo Português de Dermites de Contacto (inclui 17-butirato de hidrocortisona $0,1 \%$ e budesonida $0,1 \%$ ) e adicionalmente testes epicutâneos com betametasona creme Img/g e prednisolona em comprimido $20 \mathrm{mg}$ (macerado), ambos aplicados no dorso em oclusão durante 48 horas utilizando Finn Chambers ${ }^{\circledR}$ e com realização de duas leituras: às $48 \mathrm{~h}$ (cerca de 15 minutos após remoção de oclusão) e às 96h. Dos testes descritos, foram positivos apenas na leitura às $96 \mathrm{~h}$ para budesonida $0,1 \%(++)$. A doente fez ainda testes epicutâneos com os seus próprios produtos/ Ifármacos usados (Figura I), com aplicação diretamente na pele (dorso) e realizada oclusão durante $48 \mathrm{~h}$ utilizando Finn Chambers ${ }^{\circledR}$. Os cremes, pomada e o spray nasal foram aplicados diretamente nas Finn Chambers ${ }^{\circledR}$, os comprimidos e o pó seco dos inaladores DPI foram macerados e realizada mistura numa pequena quantidade de soro fisiológico e posteriormente aplicados nas câmaras. A primeira leitura às $48 \mathrm{~h}$ ( 15 minutos após retirar oclusão) foi negativa e a segunda e última leitura, às $96 \mathrm{~h}$, apresentou resultados positivos apenas para a associação budesonida $160 \mathrm{mcg}$ e formoterol $4,5 \mathrm{mcg}(++)$. Paralelamente, foram realizados da mesma forma testes epicutâneos com os mesmos fármacos/produtos usados pela doente, nomeadamente a associação budesonida $160 \mathrm{mcg}$ e formoterol $4,5 \mathrm{mcg}$ (DPI - pó seco), em 10 indivíduos-controlo saudáveis e foram todos negativos.

A doente realizou adicionalmente testes cutâneos por picada para aeroalergénios, que foram negativos, e estudo funcional respiratório com evidência de obstrução ligeira com reversibilidade na prova de broncodilatação.

A doente, desde então, mantém evicção de todos os produtos contendo budesonida e cumpre terapêutica inalatória alternativa a associação propionato de 


\begin{tabular}{|c|c|c|}
\hline Produto & $\begin{array}{l}\text { Resultado } \\
\text { às } 96 \mathrm{~h}\end{array}$ & \\
\hline I - Creme para dermatite seborreica & Negativo & \\
\hline 2 - Emoliente creme $\mathrm{A}$ & Negativo & $\bullet$ \\
\hline 3 - Emoliente creme B & Negativo & \\
\hline 4 - Difluocortolona + isoconazol Img/g (creme) & Negativo & \\
\hline 5 - Hidrocortisona $10 \mathrm{mg} / \mathrm{g}$ (pomada) & Negativo & \\
\hline 6 - Fluticasona $250 \mathrm{mcg}+$ salmeterol $25 \mathrm{mcg}$ inalador (DPI - pó seco) & Negativo & \\
\hline 7 - Furoato de fluticasona $27,5 \mathrm{mcg}$ (spray nasal) & Negativo & \\
\hline 8 - Budesonida $160 \mathrm{mcg}+$ formoterol $4.5 \mathrm{mcg}(\mathrm{DPI}-$ pó seco) & Positivo* (++) & \\
\hline 9 -Alprazolam 0,5mg (comprimido) & Negativo & \\
\hline 10 - Escitalopram I0mg (comprimido) & Negativo & \\
\hline II - Beta-histina I6mg (comprimido) & Negativo & \\
\hline 12 - Amlodipina 5mg + Valsartan 80mg (comprimido) & Negativo & \\
\hline 13 - Bisoprolol 5mg (comprimido) & Negativo & \\
\hline 14 - Esomeprazol 40mg (comprimido) & Negativo & $\begin{aligned} & * \text { Budesonida } 160 \mathrm{mcg} \\
+ & \text { formoterol } 4,5 \mathrm{mcg}(96 \mathrm{~h})\end{aligned}$ \\
\hline
\end{tabular}

Figura I. Produtos próprios da doente utilizados nos testes epicutâneos e respetivos resultados.

fluticasona $250 \mathrm{mcg}$ e salmeterol $25 \mathrm{mcg}$, que e para a qual tinha sido feito teste epicutâneo prévio que foi negativo.

\section{DISCUSSÃO E CONCLUSÃO}

As reações de hipersensibilidade aos corticosteroides, apesar de raras, têm vindo a ser descritas cada vez com maior frequência. Na maioria dos casos, têm sido reportados episódios de reações tardias, como na doente do caso descrito, e, portanto, habitualmente assumido o diagnóstico de hipersensibilidade tipo IV ${ }^{6}$.

A sintomatologia inicial da doente, nomeadamente dispneia, ardor orofaríngeo e edema da úvula, poderia sugerir um quadro de hipersensibilidade tipo I, mediado por IgE. Doravante, com a positividade dos testes epicutâneos, demonstrou-se que se tratava de uma reação de hipersensibilidade tipo IV, não mediada por IgE, à bu- desonida, sem reatividade com corticosteroides de outros grupos. Apesar de menos frequente, há casos descritos na literatura de reações de angioedema e urticária a fármacos que não são reações mediadas por $\lg \mathrm{E}^{7}$.

Foram realizados testes epicutâneos com a associação de budesonida $160 \mathrm{mcg}$ e formoterol $4,5 \mathrm{mcg}$ em 10 indivíduos-controlo saudáveis, que foram negativos, o que nos permitiu definir como não irritativa a concentração utilizada para o teste que se revelou positivo nesta doente. Assim, apesar de não estarem estandardizados, os testes epicutâneos com corticosteroides permitiram estabelecer um diagnóstico de hipersensibilidade tardia à budesonida e encontrar uma alternativa terapêutica.

Relativamente aos testes epicutâneos, estes não devem ser utilizados como testes de rastreio, uma vez que uma pequena percentagem de doentes pode apresentar testes cutâneos positivos sem clínica associada à sua administração - falsos positivos. Por outro lado, em doentes asmáticos que desenvolvem lesões cutâneas ou agra- 
vamento de queixas respiratórias após inalação de corticosteroides, deve ser considerada a possibilidade de se tratar de uma reação de hipersensibilidade.

O caso descrito é de particular interesse, já que as reações alérgicas a corticosteroides inalados em doentes com asma ou rinite são raros e, é um desafio na manutenção de terapêutica, dada a necessidade de utilização crónica de corticosteroides.

São poucos os casos semelhantes que se encontram descritos na literatura, mas atentando aos últimos II anos é descrito em 2008 o caso de uma mulher de 49 anos com edema da língua, disfonia e disfagia após 24 horas da administração da associação de budesonida e formoterol inalado 6 , e em 2009 são descritos dois casos refentes a uma mulher de 37 anos com angioedema labial e prurido nasal três dias após administração de budesonida nasal e uma adolescente de 15 anos com queixas de edema e prurido nasal associado a eritema facial I3h após a última administração nasal de budesonida ${ }^{8}$. Nos anos seguintes, é ainda publicado um caso de um homem de 40 anos com urticária generalizada e de uma mulher de 35 anos com angioedema dos lábios e pálpebras, ambos cerca de 8 horas após a administração de budesonida nasal ${ }^{9,10}$. Foram realizados testes epicutâneos com budesonida $(0,1 \%)$ e vários outros corticosteroides em todos estes doentes, tendo sido em todos os casos positivas as leituras das $48 \mathrm{~h}$ e $96 \mathrm{~h}$ para budesonida $(0,1 \%)$. Apenas, num caso houve testes de outros corticosteroides positivos - tixocortol ${ }^{8}$.

Mais recentemente, em 2016 é descrito o caso de uma criança de 8 anos, com quadro de urticária generalizada, recorrente, sempre após 4 horas da administração de budesonida inalada. Neste caso não foram realizados testes cutâneos, mas foi assumido o diagnóstico dado o quadro recorrente e reprodutível ${ }^{5}$.

Relativamente à doente descrita neste caso clínico, é ainda de ressalvar que além de se tratar de uma reação de hipersensibilidade rara os sintomas apresentados poderiam sugerir um caso de hipersensibilidade de tipo I. Contudo, os resultados dos testes epicutâneos demons- traram uma hipersensibilidade tipo IV restrita a budesonida (grupo B), sem reações aos principais corticosteroides de outros grupos, o que é concordante com a maioria dos casos descritos na literatura.

\section{Conflito de interesses}

Os autores declaram que não existem conflitos de interesse.

Contacto:

Sara Carvalho

Avenida Professor Egas Moniz

1649-035 Lisboa

e-mail: saracarvalhollı@hotmail.com

\section{REFERÊNCIAS}

I. Vatti RR, Ali F, Teuber S, Chang C, Gershwin ME. Hypersensitivity reactions to corticosteroids. Clin Rev Allergy Immunol 2014; 47:26-37.

2. Weiss ME, Bernstein DI, Blessing-moore J, Cox L, Lang DM, Nicklas RA et al. Drug allergy: An updated practice parameter. Ann Allergy, Asthma Immunol 2010;105:259-273.e78.

3. Basedow S, Eigelshoven S, Homey B. Immediate and delayed hypersensitivity to corticosteroids. JDDG J der Dtsch Dermatologischen Gesellschaft 2011;9:885-8.

4. Torres MJ, Canto G. Hypersensitivity reactions to corticosteroids. Curr Opin Allergy Clin Immunol 2010;10:273-9.

5. Sharma PK. Hypersensitivity with inhalational budesonide: An under recognised entity. J Clin Diagnostic Res 2016;10:FD0I-FD02.

6. Prieto García A, Tovar V, de Barrio M, Villanueva A, Tornero P. Contact allergy to inhaled budesonide*. Contact Dermatitis 2008;59:60-I.

7. Pirker C, Mišić A, Frosch PJ. Angioedema and dysphagia caused by contact allergy to inhaled budesonide. Contact Dermatitis 2003;49:77-9.

8. Pitsios C, Stefanaki EC, Helbling A. Type IV delayed-type hypersensitivity of the respiratory tract due to budesonide use: report of two cases and a literature review. Prim Care Respir J 2009;19:185-8.

9. Lopez S, Torres MJ, Antunez C, Rodríguez-Pena R, Canto G, Blanca $M$ et al. Specific immunological response to budesonide in a patient with delayed-type hypersensitivity reaction. J Invest Dermatol 2010;130:895-7.

10. Davila-Fernández G, Vazquez-Cortés S, Chamorro-Gómez M, Elices-Apellániz A. Systemic allergic reaction due to intranasal budesonide. Allergol Immunopathol (Madr) 2012;40:392-3. 